CASO CLÍNICO

\title{
Hemorragia gastrointestinal oculta, un diagnóstico retórico
}

\author{
Hidden gastrointestinal bleeding, a rhetorical diagnosis
}

Sara Sahury, ${ }^{1}$ Susana Pineda, ${ }^{2}$ Marco Sánchez. ${ }^{2}$

${ }^{1}$ Médico Residente Primer Año, Postgrado de Medicina Interna, Facultad de Ciencias Médicas, UNAH;

${ }^{2}$ Médico Internista, Sub-especialista en Gastroenterología; Servicio de Gastroenterología y Endoscopía, Departamento de Medicina Interna, Hospital Escuela; Tegucigalpa.

RESUMEN. Antecedentes: La hemorragia gastrointestinal oculta representa aproximadamente del 5\%-10\% del total de las hemorragias digestivas. Descripción del caso clínico: Paciente femenina de 62 años, procedente de Santa Cruz de Yojoa, Cortés, sin comorbilidades, con episodios intermitentes de melena de un año de evolución; evaluada y manejada con hierro parenteral y transfusiones sanguíneas. Se le realizó protocolo diagnóstico, sin encontrar origen del sangrado. Fue sometida a laparoscopía diagnóstica en junio del 2019, realizándole apendicectomía, resección yeyunal, colecistectomía y ooforectomía; cursando asintomática luego de la cirugía. En diciembre, reinicia con melena, 3-4 episodios diarios, abundante cantidad (aproximadamente $300 \mathrm{ml}$ ). Evaluada nuevamente sin lograr identificar el origen del sangrado, por lo cual se realizó vídeo capsula endoscópica que reportó, restos hemáticos sin observar sitio de sangrado. Se llevó a cabo enteroscopía de empuje y duodenoscopía observando sitio de sangrado cercano a la papila duodenal. Se decide realizar angiotomografía, la cual fue compatible con sangrado digestivo a nivel de la II y III porción del duodeno. Luego se efectúa arteriografía selectiva de vasos abdominales, evidenciando fuga de medio de contraste originada en la rama de la arteria hepática derecha de origen en la mesentérica superior. Finalmente se procedió a la aplicación de 2 hemoclip vía endoscópica obteniendo un resultado satisfactorio. Conclusiones: El tipo de lesión responsable de la hemorragia del intestino delgado depende de la edad del paciente. Los factores de riesgo de hemorragia recurrente por angioectasia incluyen el número de lesiones, la edad avanzada, comorbilidades y la terapia anticoagulante.

Palabras clave: Hemorragia gastrointestinal, Intestino delgado, Protocolos clínicos.
Recibido: 15-03-2020 Aceptado para publicación 05-01-2021

Dirección para correspondencia: Dra. Sara Daniela Sahury López.

Correo electrónico: danielasahury@gmail.com

Declaración de relaciones y actividades financieras y no financieras y conflictos de interés: ninguno.

\section{INTRODUCCIÓN}

El sangrado del intestino delgado es infrecuente y su causa, por lo general, es difícil de diagnosticar. ${ }^{1}$ Con la introducción de nuevas técnicas de imagen como la video cápsula endoscópica en 2001 y la enteroscopia profunda en 2004, se descubrió que el $75 \%$ de los pacientes previamente clasificados como con sangrado oscuro tenían fuentes de sangrado identificadas en el intestino delgado. ${ }^{2}$

En $10 \%-20 \%$ de los pacientes, no se lograba identificar el sitio de la hemorragia mediante endoscopia de vía digestiva alta (EVDA) ni colonoscopia (CLC). En esos casos el diagnóstico consignado era "sangrado de origen oscuro". Actualmente se utiliza el término "sangrado probable del intestino delgado", y su estudio es con métodos que evalúan el intestino delgado (ID) o repitiendo la endoscopia y la colonoscopia en función del escenario clínico inicial. Clínicamente, el sangrado gastrointestinal oscuro puede ser evidente con melena o hematoquezia (70\%) o puede ser oculto $(30 \%))^{3}$ La hemorragia gastrointestinal oculta también se ha denominado como "sangrado de origen intermedio". ${ }^{4}$

La hemorragia digestiva se ha clasificado según su origen en alto (SDA), si se origina por encima del ligamento de Treitz, y bajo (SDB), cuando se produce en el colon, desde el ciego hasta el ano. ${ }^{3}$ Recientemente, los conceptos tradicionales han sido revisados a la luz de los avances tecnológicos y se ha permitido establecer la reclasificación del sangrado digestivo en tres categorías esto con la finalidad de mejorar la comprensión del problema y optimizar el uso de recursos. Por definición se establece que, la hemorragia digestiva superior es aquella originada por encima de la ampolla de váter y al alcance de una gastroscopia, el sangrado digestivo medio cuya localización se ubica entre la ampolla de váter y la válvula ileocecal, es accesible a la enteroscopía profunda mediante cápsula o enteroscopía asistida por balones y el sangrado digestivo inferior evaluado por colonoscopía. ${ }^{4}$

El reporte de casos y estudios en nuestro país sobre este tema es infrecuente. Sin embargo, en muchos pacientes no se logra la identificación de la causa del sangrado y por tanto reciben un diagnóstico inadecuado y son tratados paliativamente. El protocolo de evaluación para la realización del diagnóstico es bastante laborioso, pero se ha demostrado que llegar a un diagnóstico específico tiene un valor costo efectivo, las opciones de tratamiento en nuestro medio también son limi- 
tadas debiendo ser practicadas por expertos. Los protocolos clínicos se han definido en un gran campo de investigación en el área. El objetivo de presentar este caso es evaluar los métodos diagnósticos y terapéuticos de este problema clínico tan desafiante.

\section{DESCRIPCIÓN DE CASO}

Paciente del sexo femenino; en la séptima década de la vida (62 años), analfabeta, casada, católica, ama de casa, procedente de Santa Cruz de Yojoa, Cortés. Sin antecedentes personales patológicos, quien refirió cuadro clínico caracterizado por episodios intermitentes de melena de 1 año de evolución, siendo tratada en clínica privada de Siguatepeque donde fue evaluada y diagnosticada como sangrado digestivo alto, dándose tratamiento con hierro parenteral y en ocasiones transfusiones de glóbulos rojos empacados; se realizaron estudios invasivos (endoscopia y colonoscopia) sin lograr identificar origen del sangrado por lo que se llevó a cabo laparoscopía diagnóstica y terapéutica el 29 de junio del 2019, en la cual se realizó apendicectomía, resección yeyunal, colecistectomía y ooforectomía bilateral. Luego de su cirugía se mantuvo asintomática por un período aproximado de cinco meses; en diciembre de 2019 reinicia con cuadro clínico de melena de aproximadamente 1 mes de evolución, de 3-4 episodios al día de abundante cantidad, refiere un aproximado de $300 \mathrm{ml}$. Concomitantemente refirió dolor abdominal de inicio súbito, difuso, con intensidad 4/10, que se atenuaba al reposo. Negó uso de AINES, sulfato ferroso, alcohol, tabaco, drogas o anticoagulantes. A su llegada al Hospital Escuela se le realizó historia clínica completa y examen físico con signos vitales: PA: 120/80 mmHg FC: 82 Ipm FR: 20 pm SatO $: 97 \%$. $T^{\circ}: 37^{\circ} \mathrm{C}$ Peso: $90 \mathrm{Kg}$ Glasgow:15/15, llenado capilar $<2$ segundos. Al examen físico se observó palidez en mucosa oral y conjuntival. En el abdomen se encontró blando, depresible, ruidos hidroaéreos 12 por minuto, con cicatriz en línea media de $9 \mathrm{~cm}$, no visceromegalias, no doloroso, sin signos de irritación peritoneal. Al tacto rectal se encontró con tono conservado, no se palparon masas, melena positiva; el resto del examen físico se encontró dentro de parámetros normales. Se le realizaron exámenes auxiliares que reportaron: leucocitos:4.75

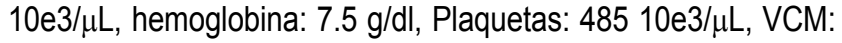
84 fL, HCM: 23.2 pg. Guayaco positivo. Bioquímica, electrolitos, pruebas de coagulación y pruebas hepáticas y renales sin alteraciones significativas. Se evalúo nuevamente con endoscopía y colonoscopía sin lograr encontrar el origen del sangrado (Figura 1), por lo que se realizó vídeo cápsula endoscópica que reportó: restos hemáticos sin observar sitio de sangrado. Se llevó a cabo enteroscopía de empuje observando sitio de sangrado cercano a la papila, inmediatamente se realizó duodenoscopía (Figura 2) que confirmo sangrado activo próximo a la papila.

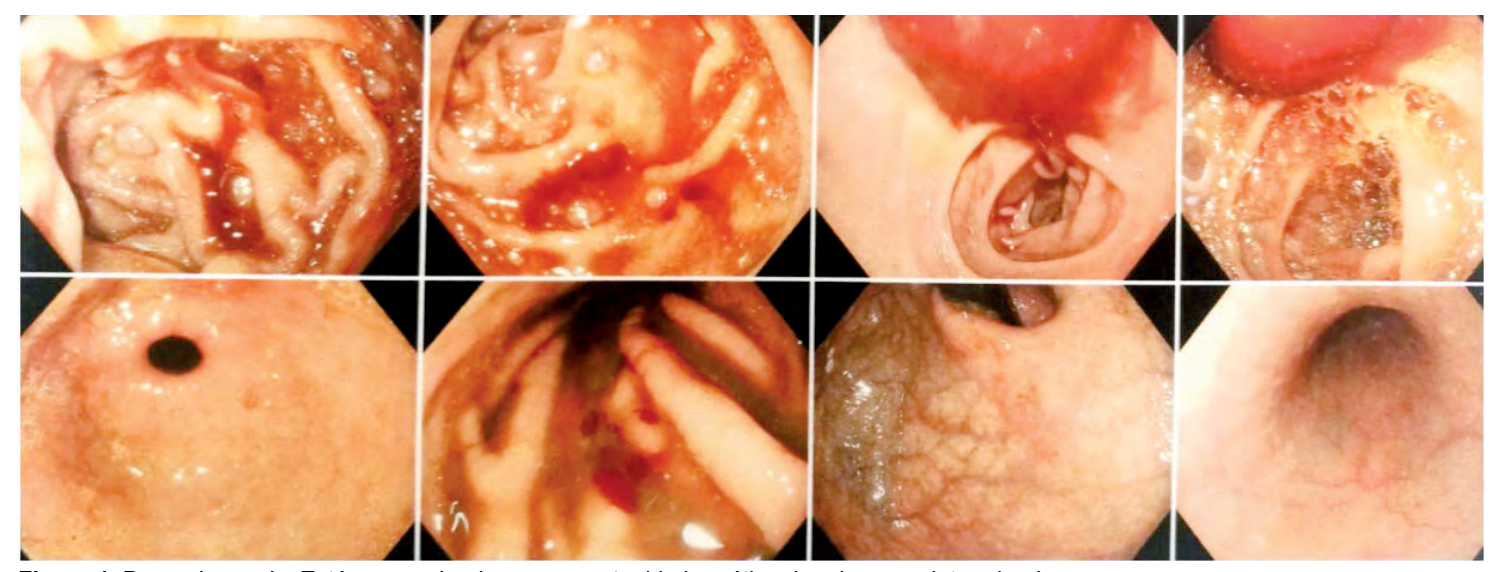

Figura 1. Panendoscopía. Estómago y duodeno con contenido hemático de origen no determinado.

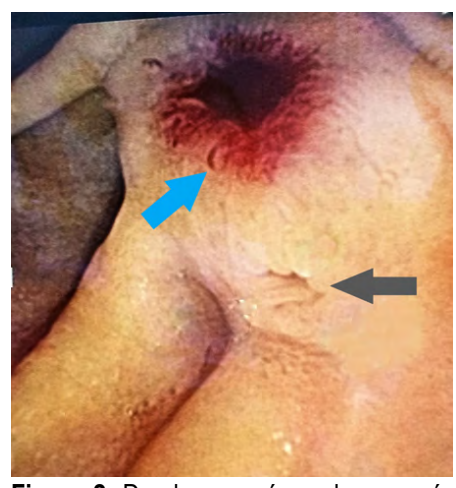

Figura 2. Duodenoscopía en la cercanía de la lesión a la papila duodenal. Se observa angiodisplasia (flecha azul) y la papila duodenal (flecha gris).

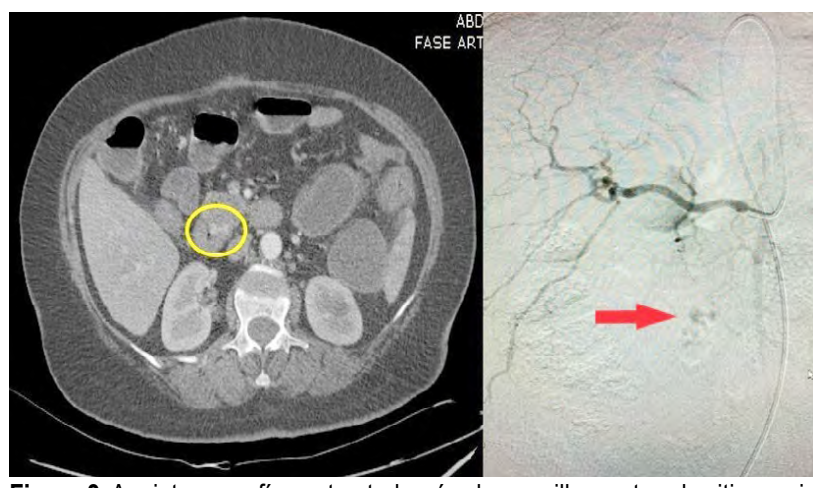

Figura 3. Angiotomografía contrastada, círculo amarillo mostrando sitio preciso de sangrado durante la adquisición en fase arterial de contraste vascular. Angiografía abdominal contrastada, se muestra el sitio de fuga del contraste (flecha roja).

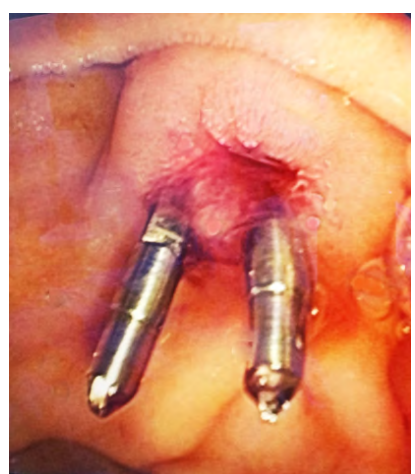

Figura 4. Colocación de hemoclips. Se coloca a $1-2 \mathrm{cms}$ de la papila duodenal, se observa flujo biliar. 
En enero 2020, se le realiza angiotomografía en fase simple y contrastada en la que se encuentran hallazgos compatibles con sangrado digestivo a nivel de la II/III porción del duodeno, quiste simple hepático y cambios osteodegenerativos; luego se le realizó arteriografía selectiva de vasos abdominales (Figura 3), observando fuga de medio de contraste a la luz intestinal originada en una rama de la arteria hepática derecha de origen en la mesentérica superior (variante anatómica), se sugirió tratamiento por cateterización supraselectiva con microcatéter, sin embargo la paciente se encontraba estable y por motivo económico no tenía posibilidad de adquirir el material necesario para realizarse este tipo de tratamiento. Este equipo no se encuentra disponible en el nivel público. Finalmente, se procedió a dar tratamiento realizando intento de aplicación de hemoclip vía endoscópica de forma fallida debido a hemorragia arterial abundante sin visibilizar el área por lo que se procede a una escleroterapia de rescate con adrenalina. En febrero 2020, se le realizo un segundo intento en el cual se logró la aplicación de 2 hemoclip aproximadamente a $2 \mathrm{~cm}$ de la papila duodenal (Figura 4), obteniendo un resultado satisfactorio. Posteriormente la paciente en mes de marzo 2020, se encontraba en buen estado general, sin signos de sangrado evidente u oculto, hemodinámicamente estable, con producción biliar normal, tolerando vía oral, con hábito defecatorio habitual normal.

\section{DISCUSIÓN}

Las causas de hemorragia de intestino delgado son múltiples e incluyen aquellas de origen vascular, inflamatorio y tumoral. Las diferentes causas varían con la edad y las zonas geográficas. En Corea del Sur, se ha encontrado como causas más frecuentes las úlceras (26\%), las angiodisplasias (10\%), las erosiones (8\%) y los tumores del intestino delgado (2\%). En los países occidentales, el $70 \%$ son de origen vascular, y las angioectasias son las más frecuentes (20\%-55\%), seguidas por los tumores (10\%-20\%) y la enfermedad de Crohn (2\%-10\%). ${ }^{3}$ En México el $85 \%$ de las hemorragias de intestino medio (HIM) son secundarias a angiodisplasias, úlceras y tumores benignos y malignos. ${ }^{5}$ En Honduras la literatura médica sobre casos de hemorragia gastrointestinal de origen medio 0 en intestino delgado es escasa. ${ }^{6}$

Las angiodisplasias son vasos sanguíneos ectásicos de paredes delgadas, dilatadas, con o sin revestimiento endotelial. Su patogénesis es incierta, pero podría estar asociada con la obstrucción crónica relacionada con el envejecimiento de las venas submucosas que se extiende a las vénulas, capilares y arterias y, en última instancia, da como resultado la pérdida de la competencia del esfínter precapilar y el desarrollo de comunicaciones arteriovenosas. ${ }^{7}$ En este caso clínico el diagnóstico resultó angiodisplasia de una rama de la arteria hepática derecha, etiología que contrasta con la clínica y la edad de la paciente.

El intestino delgado (ID), se extiende desde el piloro hasta la unión del ciego con el colon ascendente. Su longitud promedio es de 6 a $7 \mathrm{mts}$ y se encuentra subdividido en: duodeno, yeyuno e íleon. ${ }^{8}$ Debido a su extensa longitud y localización intraperitoneal, la exploración del intestino delgado conlleva gran dificultad, la reciente aparición de la cápsula endoscópica, así como la enteroscopía de doble balón ha permitido el diagnóstico y tratamiento endoscópico de la mayoría de las lesiones del intestino delgado. ${ }^{9}$ En este caso se utilizó la cápsula endoscópica lo cual contribuyo a esclarecer el diagnóstico aunque no se logró con exactitud la localización de la lesión por lo que se tuvo que recurrir a métodos adicionales.

Cuando la evaluación endoscópica estándar es negativa, como en este caso, la cápsula endoscópica y la enteroscopía con balón (simple o doble balón) anterógrada y retrógrada, son los procedimientos diagnósticos de primera línea a seguir, ambos con amplio campo de aplicación y capacidad diagnóstica equiparable..$^{10}$ La capacidad diagnóstica de la enteroscopía es del $73 \%$ cuando es precedida por una vídeo cápsula endoscópica (VCE) positiva ${ }^{4}$. Los resultados de este caso apuntan a que ambos métodos se complementan y que la VCE como primera estrategia permite guiar la vía de abordaje. ${ }^{4} \mathrm{~A}$ pesar de las dificultades con el equipo se logró seguir el protocolo clínico recomendado por la Asociación Americana de Gastroenterología (AGA) para llegar a un diagnóstico específico. ${ }^{11}$

La video-cápsula endoscópica (VCE) es un estudio que permite la evaluación de la mucosa del intestino delgado (ID) de forma mínimamente invasiva y con una baja tasa de eventos adversos. ${ }^{12,13}$ Consiste en un dispositivo de $26 \mathrm{~mm}$ de largo $x$ $11 \mathrm{~mm}$ de ancho y pesa $3,7 \mathrm{~g}$ de peso. Contiene una pequeña videocámara, una fuente de iluminación formada por cuatro diodos que emiten luz blanca (LED), un sistema de transmisión de imágenes, una pequeña batería que dura aproximadamente 8 horas y una antena, todo ello incluido en el interior de una funda de plástico biocompatible. Almacena todas las imágenes en un microchip y está diseñado para ofrecer una vista panorámica de $360^{\circ}$ con tecnología sin cables. La ampliación que ofrece es de 1:8 y el tamaño mínimo de detección de $0,1 \mathrm{~mm} \cdot{ }^{14} \mathrm{Al}$ ingresar al tracto digestivo, la cápsula endoscópica adquiere las imágenes a una velocidad de 2 a 6 imágenes por segundo ${ }^{15}$. Finalmente se obtienen en promedio un total de 55000 imágenes en un período de 8 a 20 horas, que son analizadas por el clínico a través del software. La principal desventaja que ofrece el uso de la cápsula endoscópica es que es incapaz de realizar toma de biopsias o intervenciones terapéuticas. ${ }^{16}$ En este caso se utilizó una pillcam y no se observaron complicaciones y/o efectos adversos, se evaluó detenidamente el vídeo por los especialistas sin lograr una ubicación exacta de la hemorragia gastrointestinal debido a que la cápsula no tiene la opción de detenerse en un lugar determinado por más tiempo del programado en cada software, además la hemorragia abundante impidió que se ubicara con exactitud la lesión. Cabe destacar que en Honduras el uso de la VCE es limitado por el costo de este método, el cual no está disponible a nivel público.

La retención de la VCE es la complicación más frecuente, definida como la persistencia de la VCE en el intestino delgado durante más de 2 semanas. ${ }^{14}$ La Sociedad Europea de Endoscopía Gastrointestinal (ESGE) recomienda que, en pacientes con hemorragia gastrointestinal oscura manifiesta, se realice una endoscopía con cápsula de intestino delgado tan pronto como sea posible después del episodio de hemorragia, de manera óptima dentro de los 14 días, para maximizar el rendimien- 
to diagnóstico. ${ }^{17}$ Debido a que la paciente no tenía accesibilidad en el tiempo recomendado se realizó la VCE un mes luego del reinicio de los síntomas obteniendo diagnóstico de hemorragia gastrointestinal de ubicación poco clara.

En pacientes con sospecha de hemorragia gastrointestinal, la clasificación de Saurin se ha recomendado para la evaluación de la relevancia de las lesiones. Según la clasificación de Saurin, las lesiones detectadas en la cápsula endoscópica se clasifican como P0, P1 y P2. Las lesiones P0 son aquellas que no tienen potencial de sangrado, incluidas las venas submucosas visibles, los divertículos sin presencia de sangre o los nódulos sin ruptura de la mucosa. Las lesiones $\mathrm{P} 1$ son aquellas que se considera que tienen un potencial hemorrágico incierto, como manchas rojas en la mucosa intestinal o erosiones pequeñas 0 aisladas. Las lesiones P2 son aquellas que se considera que tienen un alto potencial de sangrado, como los angiomas típicos, las ulceraciones grandes, los tumores o las várices. En este caso se clasificaría como un P2. Para otras indicaciones además de la hemorragia gastrointestinal, hace falta una clasificación universalmente aceptada. ${ }^{17}$

En este caso clínico se realizó también enteroscopía por pulsión diagnóstica o enteroscopía de empuje; este es un examen endoluminal transoral del yeyuno proximal utilizando un endoscopio largo y flexible. Alcanza más allá del ángulo de Treitz $(150-160 \mathrm{~cm})$. Su eficacia en el diagnóstico de una hemorragia de intestino delgado varía entre el 24 y el $56 \%$, con escasa tasa de complicaciones. Sólo permite la exploración del yeyuno proximal. Permite el muestreo de tejido y la aplicación de soluciones terapéuticas para la lesión sangrante.,15 La gran ventaja de esta modalidad consiste en la posibilidad de realizar terapéutica endoscópica. ${ }^{4}$ En Honduras no se cuenta con el equipo adecuado para realizar enteroscopía, sin embargo, se utilizó el equipo disponible de manera que se pudiera alcanzar la longitud deseada del intestino delgado.

Los estudios de radiología son complementarios a los procedimientos endoscópicos. La angiotomografía se realiza mediante contraste intravenoso, no requiere distensión intestinal y puede detectar tasas de sangrado tan bajas como $0.3 \mathrm{ml} /$ min. Además, ayuda a identificar el posible sitio de sangrado y proporciona orientación para un manejo adicional, como la angiografía con embolización o cirugía. ${ }^{18}$ En este caso se realizó una angiotomografía simple y una contrastada donde muestra con exactitud el vaso aberrante en la paciente.

En individuos sanos, durante el proceso digestivo del bolo alimenticio se pierden 0,5-1,5 mg de hemoglobina por gramo de heces, lo que equivale a una pérdida digestiva de $0,25-0,75 \mathrm{mg}$ de hierro elemental al día. ${ }^{2}$ El manejo inicial de la paciente fue con hierro parenteral y transfusiones de glóbulos rojos empacados, siendo tratada como una hemorragia digestiva alta.

El tratamiento de las angiodisplasias es difícil, sin importar las causas, y faltan ensayos aleatorizados y controlados para guiar la terapia. El manejo implica atención de apoyo, con suplementos de hierro, transfusión de sangre cuando esté indicado y terapia endoscópica cuando sea posible. ${ }^{7}$ En este caso se realizó la aplicación de 2 hemoclips obteniendo un resultado satisfactorio hasta la fecha.
La terapia endoscópica incluye coagulación con plasma de argón, colocación de hemoclips endoscópicos, terapia térmica de contacto (electrocauterio bipolar o sonda de calentamiento), o combinando estas técnicas. También, se puede usar una inyección de solución salina normal o epinefrina como complemento de una de estas terapias. ${ }^{12}$ Desde 2001, la coagulación con plasma de argón se ha utilizado principalmente como el tratamiento de elección endoscópico. ${ }^{11}$ La mayoría de estos tratamientos en nuestro país desafortunadamente solo está disponible a nivel privado.

Los pacientes con hemorragia clínica activa e inestabilidad hemodinámica deben ser tratados con angiografía convencional, con embolización o infusión de vasopresina. ${ }^{12}$ En Honduras este tratamiento tiene un costo muy elevado y requiere especialistas altamente entrenados en esta área, los cuales actualmente son escasos, lo que hace que esta posibilidad de tratamiento este lejos de la realidad de los pacientes aun cuando el paciente se encuentre en estado crítico.

Estudios recientes no recomiendan en definitiva el tratamiento farmacológico, algunos estudios afirman que debe quedar reservado para aquellos pacientes que no han respondido a los tratamientos endoscópicos o como medida paliativa. Esta modalidad se basa en el uso de fármacos con capacidad para actuar sobre lesiones vasculares, mejorando estabilidad del endotelio 0 minimizando la angiogénesis. Se han utilizado hormonas (estrógenos con o sin progesterona), análogos de la somatostatina, eritropoyetina y factor de Von Willebrand, pero no parece que tengan utilidad a largo plazo en pacientes con sangrado manifiesto. La talidomida y el octeótrido han demostrado tener algún beneficio. Se han realizado ensayos de casos prometedores en pacientes con telangiectasia hemorrágica hereditaria tratados con bevacizumab (antagonista del factor de crecimiento endotelial vascular), que parece disminuir los requerimientos transfusionales, pero se necesitan más estudios para confirmarlo. ${ }^{19}$ En este caso clínico no se consideró el uso de fármacos para el tratamiento.

Se han estimado implicaciones económicas que existen entre los sangrados digestivos altos y bajos (colónicos e intestinales) y se concluyó que el subgrupo intestinal requiere, en general, prolongadas hospitalizaciones y múltiples procedimientos diagnósticos, por lo que genera mayores $\operatorname{costos}^{1}$ como se observa en este caso.

Basándonos en este caso clínico hacemos las siguientes recomendaciones: fomentar el reporte de casos en el tema para enriquecer el conocimiento en cuanto a esta patología poco frecuente y así obtener datos estadísticos relevantes en nuestro país; brindar el protocolo diagnóstico adecuado cuando la situación lo amerite y sea económicamente posible; dar seguimiento a estos casos para verificar la efectividad de los tratamientos disponibles en nuestro país.

\section{CONTRIBUCIONES}

SS, análisis e interpretación de datos, encargada de la redacción final del manuscrito. SP, concepción y diseño del estudio; adquisición de información y revisión crítica del contenido intelectual importante. MS, adquisición de información y revisión crítica del contenido intelectual importante. Se aprobó de mane- 
ra unánime la versión a ser publicada; y estamos de acuerdo en ser considerados responsables de todos los aspectos del trabajo, asegurando que las preguntas relacionadas a la exactitud $o$ integridad de cualquier parte del trabajo sean adecuadamente investigadas y resueltas.

\section{AGRADECIMIENTOS}

Se reconoce al Dr. Carlos Rivera por su contribución en la interpretación de imágenes radiológicas en este caso.
1. Travis A, Saltzman J. Sangrado del intestino delgado: Causas, diagnóstico y tratamiento. [Internet]. Bethesda: American College of Gastroenterology; 2017. [citado 23 mayo 2020]. Disponible en: https://gi.org/patients/ recursos-en-espanol/sangrado-del-intestino-delgado-causas-diagnostico$y$-tratamiento/

2. Robles L, Pérez-Mateo Regadera M. Hemorragia gastrointestinal oculta. En: Montoro MA; García Pagán JC, editores. Libro de Gastroenterología y Hepatología: problemas comunes en la práctica clínica. $2^{\mathrm{a}}$ ed. Madrid: Servicio de Aparato Digestivo, Hospital General Universitario, Alicante. Asociación Española de Gastroenterología; 2012,p.81-90

3. Sandoval Riveros C, Lúquez Mindiola A, Fernández H, Otero Regino W. Sangrado del intestino delgado: enfoque y tratamiento. Rev Colomb Gastroenterol [Internet]. 2017[citado 23 mayo 2020];32(3):245-57. Disponible en: https://revistagastrocol.com/index.php/rcg/article/view/156

4. Casanova R. Enfoque diagnóstico del sangrado digestivo oscuro. Gen [Internet]. 2013[citado 23 mayo 2020];67(3):175-180. Disponible en: http://ve.scielo.org/scielo.php?script=sci_arttext\&pid=S0016$35032013000300011 \& \operatorname{lng}=\mathrm{es}$.

5. García-Compean D, Jáquez-Quintana JO, González-González JA, Maldonado-Garza HJ. Hemorragia de intestino medio causada por uncinariasis y diagnosticada por cápsula endoscópica. Caso clínico. Rev Gastroenterol Mex [Internet]. 2013[citado 13 abril 2020];78(3):196-197. Disponible en: DOI: 10.1016/j.rgmx.2013.03.002

6. Durán-López CA, Girón F. Lesión de Dieulafoy en el yeyuno: presentación de caso y revisión de la literatura. Rev Cienc Forenses Honduras [Internet]. 2019[citado 13 abril 2020];5(1):14-20. Disponible en: https://www. lamjol.info/index.php/RCFH/article/view/8680/9877

7. Brock AS, Cook JL, Ranney N, Rockey DC. Clinical problem-solving. A not-so-obscure cause of gastrointestinal bleeding. N Engl J Med [Internet]. 2015[citado 14 marzo 2020];;372(6):556-61. doi: 10.1056/NEJMcps1302223.

8. Ferrufino JC, Taxa L, Angeles G. Histología normal del intestino delgado. Rev Med Hered [Internet]. 1996[citado 14 marzo 2020];7(1):46-57. Disponible en: http://www.scielo.org.pe/scielo.php?script=sci_arttext\&pid=S1018130X1996000100009\&ing=es.

9. Serrano León MD, Rodríguez Pardo MJ. Protocolo diagnóstico de la hemorragia digestiva de origen oscuro. Medicine. Programa de Formación Médica Continuada Acreditado [Internet]. 2012[citado 14 marzo 2020];11(4):249-252. DOI: 10.1016/S0304-5412(12)70294-9

10. Gonzales $\mathrm{C}$, Osorio H, Melgar C. Hemorragia digestiva de origen oscuro: una causa no sospechada. RFS, Revista Facultad de Salud [Internet]. 2015[citado 14 marzo 2020];7(2):57-60. Disponible en: https://journalusco. edu.co/index.php/rfs/article/view/957

11. Gerson LB, Fidler J, Cave DR, Leighton JA. ACG Clinical Guideline: diagnosis and management of small bowel bleeding. Am J Gastroenterol [Internet]. 2015[citado 21 marzo 2020];110(9):1265-1287. Disponible en: https://journals.Iww.com/ajg/Fulltext/2015/09000/ACG_Clinical_Guideline_Diagnosis_and_Management.10.aspx

12. Gompertz $\mathrm{M}$, Díaz $\bar{C}$, Gil L. Estudio de intestino delgado: experiencia en el uso de Cápsula Patency en la prevención de retención de Video-cápsula Endoscópica. Gastroenterol latinoam [Internet]. 2019[citado 21 marzo 2020];30(3):129-134. Disponible en: https://gastrolat.org/estudio-de-intestino-delgado-experiencia-en-el-uso-de-capsula-patency-en-la-prevencionde-retencion-de-video-capsula-endoscopical

13. Lynch Mejía M. La cápsula endoscópica como estudio diagnóstico en gastroenterología. Revista Médica Sinergia [Internet]. 2019[citado 21 marzo 2020];4(4):18-25. Disponible en: https://doi.org/10.31434/rms.v4i4.179

14. Higuera M, Castellano S, Navajas S, García L. Protocolo de indicaciones de la video cápsula endoscópica. Medicine - Programa de Formación Médi- ca Continuada Acreditado [Intenet]. 2020[citado 21 marzo 2020];13(1):5962. Disponible en: https://doi.org/10.1016/j.med.2020.01.009

15. Pennazio M, Spada C, Eliakim R, Keuchel M, May A, Mulder CJ, et al. Small-bowel capsule endoscopy and device-assisted enteroscopy for diagnosis and treatment of small-bowel disorders: European Society of Gastrointestinal Endoscopy (ESGE) Clinical Guideline. Endoscopy [Internet]. 2015[citado 14 marzo 2020];:47(4):352-76. DOI http://dx.doi.org/ 10.1055/ s-0034-1391855

16. Varela Lema L, Puñal Riobóo J, Ruano Raviña A. Utilidad clínica de la cápsula endoscópica en el sangrado gastrointestinal de original de origen oscuro [Internet]. Madrid: Ministerio de Sanidad y Consumo; 2006. [citado 21 marzo 2020]. Disponible en: https://www.sergas.es/Docs/Avalia-t//nfCapEndoscop.pdf

17. Spada C, McNamara D, Despott E, Adler S, Cash B, Fernández I, et al. Performance measures for small-bowel endoscopy: a European Society of Gastrointestinal Endoscopy (ESGE) Quality Improvement Initiative. Endoscopy [Internet] 2019[citado 5 abril 2020]; 51(06): 574-598. DOI: 10.1055/a-0889-9586

18. Baez-Corujo F, Class-Vázquez W, Martínez-Souss J. Small Bowel Bleeding: A Challenging Diagnosis.PR Health Sci J[Internet]. 2019[citado 21 marzo 2020];38(2):122-124. Disponible en: https://www.ncbi.nlm.nih.gov/ pubmed/31260559

19. Casado-caballero F, Moral-Martínez M, Berenguer-Guirado R. Actitud ante el paciente con hemorragia digestiva de origen oscuro. RAPD online [Internet]. 2015[citado 21 marzo 2020]; 38(5):214-225. Disponible en: https:// www.sapd.es/revista/2015/38/5/03

ABSTRACT. Background: Hidden gastrointestinal bleeding represents approximately $5 \%-10 \%$ of total gastrointestinal bleeding. Clinical case description: 62 years old, female patient from Santa Cruz de Yojoa, Cortés. Without comorbidities, who reports intermittent episodes of mane of 1 year of evolution. Evaluated and managed with parenteral iron and blood transfusions. Studies were performed, without finding the source of the bleeding. She underwent diagnostic laparoscopy in June 2019, performing an appendectomy, jejunal resection, cholecystectomy, and oophorectomy; staying asymptomatic after surgery. In December, restarts with mane, 3-4 episodes daily, abundant quantity, (approximately $300 \mathrm{ml}$ ). Evaluated again without being able to identify the origin of the bleeding, for which endoscopic video capsule was performed, that reported hematic remains without observing the bleeding site. A push enteroscopy and a duodenoscopy were performed, observing a bleeding site close to the duodenal papilla. It was decided to perform angiotomography, which was compatible with digestive bleeding at the level of the II and III portion of the duodenum. Then a selective arteriography of abdominal vessels is performed, showing leakage of contrast medium originating in the right hepatic artery of origin in the superior mesenteric. Finally, 2 hemoclips were applied endoscopically, obtaining a satisfactory result. Conclusions: The type of lesion responsible for small bowel bleeding depends on the age of the patient. Risk factors for recurrent bleeding from angioectasia include number of lesions, advanced age, comorbidities, and anticoagulant therapy. Keywords: Clinical Protocols, Gastrointestinal Hemorrhage, Small intestine. 